\title{
Ablation of mouse adult neurogenesis alters olfactory bulb structure and olfactory fear conditioning
}

\author{
Matthew T. Valley ${ }^{1}$, Tanner R. Mullen', Lucy C. Schultz' ${ }^{1}$ Botir T. Sagdullaev ${ }^{2}$ and Stuart Firestein ${ }^{1 *}$ \\ Department of Biological Sciences, Columbia University, New York, NY, USA \\ Weill Medical College of Cornell University, Burke Medical Research Institute, White Plains, NY, USA
}

Edited by:

Pierre M. Lledo, Pasteur Institute,

France

\section{Reviewed by:}

Luca Bonfanti, University of Turin, Italy

Pierre M. Lledo, Pasteur Institute,

France

*Correspondence:

Stuart Firestein, Department of Biological Sciences, 600 Fairchild,

Columbia University, New York, NY, 10027, USA.

e-mail: sjf24@columbia.edu
Adult neurogenesis replenishes olfactory bulb $(\mathrm{OB})$ interneurons throughout the life of most mammals, yet during this constant flux it remains unclear how the OB maintains a constant structure and function. In the mouse $\mathrm{OB}$, we investigated the dynamics of turnover and its impact on olfactory function by ablating adult neurogenesis with an $x$-ray lesion to the subventricular zone (SVZ). Regardless of the magnitude of the lesion to the SVZ, we found no change in the survival of young adult born granule cells (GCs) born after the lesion, and a gradual decrease in the population of GCs born before the lesion. After a lesion producing a $96 \%$ reduction of incoming adult born GCs to the OB, we found a diminished behavioral fear response to conditioned odor cues but not to audio cues. Interestingly, despite this behavioral deficit and gradual anatomical changes, we found no electrophysiological changes in the GC population assayed in vivo through dendro-dendritic synaptic plasticity and odor-evoked local field potential oscillations. These data indicate that turnover in the granule cell layer is generally decoupled from the rate of adult neurogenesis, and that $\mathrm{OB}$ adult neurogenesis plays a role in a wide behavioral system extending beyond the OB.

Keywords: granule cell, sub-ventricular zone, irradiation, amygdala, freezing, dendro-dendritic, survival, plasticity

\section{INTRODUCTION}

Adult neurogenesis, the process whereby new neurons are born, survive and integrate into adult neural circuits, offers the potential for plasticity and repair in the mature brain. It is a prominent feature of the olfactory bulb (OB), olfactory epithelium, and dentate gyrus, however, the regulation of this process and its functional significance to the organism remains unclear.

Adult born neurons destined for the $\mathrm{OB}$ originate from a population of astrocyte stem cells lining a portion of the subventricular zone (SVZ) and rostral migratory stream (RMS) (Alvarez-Buylla and Garcia-Verdugo, 2002; Bonfanti and Peretto, 2007). Neuroblasts migrate from their germinal niche through the RMS into the $\mathrm{OB}$ where a most of them migrate toward lamina of the granule cell layer (GCL) to become GCs (Carleton et al., 2003), and a minority migrate towards the glomerular layer to become peri-glomerular interneurons (Belluzzi et al., 2003). Recent work on the maturation of adult born GCs indicates that they first form basal synaptic connections with centrifugal fibers (Whitman and Greer, 2007; Kelsch et al., 2008), followed by formation of a dendro-dendritic synapse with mitral cells (MC) (Carleton et al., 2003). Once fully integrated into the circuit, GCs integrate olfactory input from the periphery with diverse inputs from higher brain areas (Shepherd, 1998), and transmit this activity towards MC through dendro-dendritic GABA release (Jahr and Nicoll, 1980).

Theories regarding the computational function of $\mathrm{OB}$ adult neurogenesis often posit that by providing a continuously refreshed population of synaptic connections, young neurons may enable the system to better adapt to new olfactory environments (Cecchi et al., 2001; Lledo et al., 2006). There are three lines of evidence in support of this. First, the rate of neural proliferation in the SVZ appears to be sensitive to conditions with unique olfactory demands such as pregnancy (Shingo et al., 2003), and pheromone exposure (Mak et al., 2007). Next, between 2 and 3 weeks after genesis, adultborn neurons undergo massive cell death that is negatively correlated with olfactory activity (Petreanu and Alvarez-Buylla, 2002; Rochefort et al., 2002; Saghatelyan et al., 2005; Yamaguchi and Mori, 2005) and is sensitive to afferent activity from higher brain regions (Alonso et al., 2006; Mouret et al., 2008). This may reflect a mechanism that selects which young neurons survive, and thus which new connectivity patterns are added to the circuit. Finally, adult born GCs in the OB are functionally distinct from the GCs born during development (Lemasson et al., 2005), and young cells at any age express unique plasticity in their synapses with centrifugal fibers (Gao and Strowbridge, 2009; Nissant et al., 2009). Together, these data suggest that adult neurogenesis is not merely a deregulated form of post-natal development, but likely serves a critical function.

Recent work has shown that after ablation of SVZ adult neurogenesis and subsequent cell loss in the GCL, there are no behavioral deficits in olfactory discrimination, odor detection thresholds, or in the retention short-term olfactory memory (Imayoshi et al., 2008; Lazarini et al., 2009). However, there are conflicting reports regarding the importance of adult neurogenesis to long-term olfactory memory (Imayoshi et al., 2008; Lazarini et al., 2009). In general, the functional impact of adult neurogenesis in the $\mathrm{OB}$ is still unclear.

In this study we find that a chronic loss of adult neurogenesis impacts both $\mathrm{OB}$ structure and olfactory behavior, but not OB physiology. We ablated SVZ neurogenesis and characterized a consequent gradual reduction in the size of the GCL. We also 
observed that the survival of young GCs is insensitive to the rate of neurogenesis, suggesting a general decoupling between neuronal survival in the $\mathrm{OB}$ and neurogenesis in the SVZ. Functionally, we observed a behavioral disruption in olfactory-cued fear conditioning, but surprisingly this behavioral phenotype did not correlate with any alteration in GCL physiology. This suggests that adult neurogenesis functions in a behavioral system extending beyond the OB.

\section{MATERIALS AND METHODS ANIMALS}

All mice were C57/BL6j purchased from the Jackson Laboratories, and housed at Columbia University in accordance with institutional I.A.C.U.C standards for animal care. Animals were group housed, and left to recover between 3 days and 2 weeks after arrival, before beginning the $\mathrm{x}$-ray irradiation. All animals were between 8 and 12 weeks old at the time of irradiation. Therefore, all mice in this study were between 3 and 8 months old at the time of testing. For behavioral testing, mice were male $\mathrm{C} 57 \mathrm{Bl} / 6 \mathrm{~J}$ irradiated at 6-8 weeks of age and were naïve to behavioral testing prior to fear conditioning. Animals were handled for approximately 1 min once daily for 2 days prior to experimentation to minimize handling induced stress during the experiment. Mice were housed in groups of five and sustained on a 12-h light/dark cycle with ad lib access to food and water.

\section{X-RAY IRRADIATION}

The X-ray source for all experiments was a Siemans Stabilopan operated at $12 \mathrm{~mA}$ and $300 \mathrm{kVp}$ with a $0.2-\mu \mathrm{m}$ Copper filter to harden the beam, and emitted $30 \mathrm{~cm}$ above the head of the mouse. The ionization dosage to water was measured by the Department of Radiation Oncology Physics, and the corrected real dosage was calculated previously to be approximately 1.8 Gy per minute (Santarelli et al., 2003). Therefore, a full 15 Gy dosage consisted of a 2:40 min exposure given three times within a week. The mouse was anesthetized with an intraperitoneal injection of ketamine $(100 \mathrm{mg} / \mathrm{kg})$ and xylazine $(7 \mathrm{mg} / \mathrm{kg})$, and mounted in a stereotaxic frame (Kopf, Tujunga, CA, USA). A lead box was placed over the mouse, and aligned to the ear bars of the stereotaxic frame. One-half inch thick lead shielding covered the entire length of the mouse, except for a window $5 \mathrm{~mm}$ (rostral-caudal) by $13 \mathrm{~mm}$ (medial-lateral) that could be moved along the rostral-caudal axis of the animal. This sliding window could be positioned relative to the ear bars with the aid of a ruler machined into the lead box ensuring precise positioning of the window between animals and between $\mathrm{x}$-ray sessions. For sham irradiated animals, cagemates were anesthetized at the same time and locations as their irradiated counterparts.

\section{HISTOLOGY}

Mice were transcardially perfused with $4 \%$ paraformaldehyde in PBS, and their dissected brain was post-fixed in $4 \%$ paraformaldehyde for an additional $24 \mathrm{~h}$ at $4^{\circ} \mathrm{C}$. Brains were cryoprotected in $40 \%$ sucrose solution, and frozen in a cutting mold in OCT. Twenty micrometer thick sections were made using a freezing cryostat and then were mounted directly to glass slides (Fischer Superfrost Plus, Pittsburgh, PA, USA).

\section{IMMUNOHISTOCHEMISTRY}

Cryostat frozen section cut at $20-\mu \mathrm{m}$ thickness were treated for antigen retrieval by incubating in $0.01 \mathrm{M} \mathrm{Na}$-citrate for $10 \mathrm{~min}$ in a conventional kitchen steamer. The following primary antibodies were used: mouse-anti-BrdU (1:100, BD Biosciences, San Josa, CA, USA), rat-anti-BrdU (1:1000, Accurate Chemical, Westbury, NY, USA), donkey anti-doublecortin (1:000, Dcx C-18, Santa Cruz Biotechnology, Santa Cruz, CA, USA). Secondary antibodies raised in donkey were used at 1:750 (Alexa 488 and Alexa 594, Invitrogen, Eugene, OR, USA), or biotinylated secondary antibodies were used (donkey anti-rat and biotinylated donkey anti-goat, 1:1000, Jackson ImmunoResearch, West Grove, PA, USA) followed by streptavadin conjugated Cy-3 (1:1000, Zymed, San Franscicso, CA, USA). Counterstain was performed using DAPI or Hoescht dyes.

\section{CELL COUNTING}

BrdU counts of the SVZ were made from coronal sections and involved complete counting of all cells along the lateral wall of the SVZ. Sections of the SVZ were cut every $120 \mathrm{um}$ with the rostralmost slice aligned to the rostral-tip of the SVZ. Eighty slices caudal to this point were counted, and the total number of cells across all sections was taken to represent the proliferative state of the tissue. Counting was done under $20 \times$ epiflourescence, and was done blind to sample identity.

For BrdU counts in the main $\mathrm{OB}$, complete counts were made from coronal sections made every $120 \mathrm{um}$ anterior to the closure of the AOB. The area of each section was measured, and the cell count was then normalized to this area to obtain the surface density of BrdU nuclei. We chose to make this normalization to eliminate variability caused by variations in the plane of sectioning.

\section{VOLUME ESTIMATION}

Olfactory bulbs were sectioned into $60-\mu \mathrm{m}$ sagittal sections using a cryostat. On chilled slides, these sections were suspended in a drop of PBS to prevent folding and compression artifacts, and were then dehydrated on a heating plate. Cell nuclei were stained using hoescht or DAPI stain, and photographed for off-line analysis. Total volume was calculated by multiplying the total section thickness $(60 \mu \mathrm{m})$ by the surface area of every section (Cavalieri's principle).

\section{DENSITY ESTIMATION}

We developed a method to use the optical dissector off-line, eliminating the need to count cells while on a confocal microscope. Confocal z-stacks of DAPI-stained nuclei were taken every $1 \mu \mathrm{m}$ over the middle $16 \mu \mathrm{m}$ of a $20 \mu \mathrm{m}$ cryosection on a laser-scanning confocal microscope (Zeiss LSM510, Thornwood, NY, USA). Care was taken to maintain similar brightness for images at each depth. The middle $12 \mu \mathrm{m}$ of each $\mathrm{z}$-stack was then false-colored off-line using the program ImageJ, whereby the top two slices were colored red, and the bottom $10 \mu \mathrm{m}$ slices were colored green to blue (see Figure 3E). In this way we could easily define cells within an exclusion volume by identifying all red, yellow or orange cells. The remaining cells, if not occupying this exclusion volume, would be colored blue-green. For each anatomical counting region, the number of cells in the exclusion volume and in the counting volume was calculated at three randomly chosen slices anterior to the closure of the AOB. 


\section{ODOR DELIVERY}

A custom made olfactometer was adapted from a previous design (Lorig et al., 1999) to deliver odors during electrophysiology. Compressed air was humidified and passed by the animal's nose at a rate of $11 / \mathrm{min}$ (carrier stream). The odor amyl acetate, to which the animals were naïve, was dissolved in $1 \mathrm{ml}$ DMSO to a final concentration of $0.5 \mathrm{mM}$, and a $20 \mathrm{ml}$ vapor headspace was kept saturated with a cotton or paper wick to maximize liquid surface area. Amyl acetate was used as a representative odor because it evoked highly reproducible and stereotyped spectral-temporal patterns across multiply sampled positions within the GCL. Odor puffs, 2 s in duration, were diverted into the carrier stream, and puffs were separated by a minimum of $40 \mathrm{~s}$. To avoid pressure changes, a compensatory open air channel was switched off during odor presentation. No decrease of odor response was measured in the LFPs after repeated stimulation over hours (unpublished observations). Odor concentration reported in the text does not take into account the dilution of the saturated headspace into the carrier stream, therefore final concentrations are much less. For every mouse, odor was delivered at least seven times, spaced apart with pulses of solvent headspace.

\section{ELECTROPHYSIOLOGY}

Anesthesia was induced with an intraperitoneal injection of ketamine $(100 \mathrm{mg} / \mathrm{kg})$ and xylazine $(7 \mathrm{mg} / \mathrm{kg})$ in mammalian Ringer's solution and supplemental injections were given every $\sim 45 \mathrm{~min}$. The animal was mounted in a stereotaxic frame (David Kopf Instruments, Tujunga, CA, USA) and body temperature was maintained at $37^{\circ} \mathrm{C}$ with a feedback controlled heating pad (CT1000, CWE, Ardmore, PA, USA). The animal's nose was inserted into an air-tight gas mask through which humidified air from the olfactometer passed at a rate of $1 \mathrm{l} / \mathrm{min}$. Two small craniotomies were performed at the left hemisphere at stereotaxic coordinates corresponding to the region above the LOT, and to the central dorsal surface of the $\mathrm{OB}$. A custom made tungsten recording electrode (Hubel, 1957) with an impedance of 0.7-2 M $\Omega$ was inserted $1 \mathrm{~mm}$ deep with a hydraulic microdrive into the GCL of the MOB, and a custom made bipolar tungsten stimulating electrode was driven between 3.5-4.5 $\mathrm{mm}$ deep into the LOT. We recorded evoked field potentials (Figure 5) from the same location in the GCL as for the LFP recordings (Figure 6). Correct placement of the stimulating electrode was confirmed by recording the antidromic evoked waveform in the GCL (Shepherd and Haberly, 1970). If the evoked GCL waveform did not contain three distinct phases corresponding to (1) back-propagating spikes (2) field GC excitatory postsynaptic potentials (EPSPs) and (3) field MC inhibitory postsynaptic potentials (IPSPs), we assumed the stimulation electrode was reaching too many centrifugal afferents, and not enough LOT fibers. In this case the stimulating electrode was inserted to a new position. In some animals the position of the stimulating and recording electrode was confirmed histologically after burning the tip position, and visualized in $100-\mu \mathrm{m}$ thick vibratome slices with cresyl violet.

Local field potentials were amplified (DP-301, Warner Instruments, Hamden, CT, USA), filtered (1 Hz highpass, $3 \mathrm{kHz}$ lowpass), and digitized at $5-10 \mathrm{Khz}$ (Power 1401, CED, UK). Breathing was continuously monitored with a piezoelectric force-transducer (Stoelting, Wood Dale, IL, USA) in contact with the animal's back, and this signal was used to trigger odor delivery upon crossing a threshold corresponding to the transition of inhalation to exhalation (I/E transition). In the breathing trace, inhalation occurs during positive values; exhalation during negative values (see Figure 6B). All on-line event detection and triggering was done using the software Spike2 (CED, UK). Similarly, LOT stimulation was triggered by the phase of the breathing cycle, and current was created using a pulse generator and stimulus isolation unit (Grass S88, W. Warwick, RI, USA). For every mouse, the stimulus-intensity response relationship was recorded with the GCL recording electrode. All subsequent stimulation in the paired-pulse paradigm was given at saturating intensity $(1-1.5 \mathrm{~mA}$ over $100 \mu \mathrm{s})$. Experiments that involved LOT stimulation were always performed after the completion of odor delivery protocols.

\section{LFP SIGNAL PROCESSING AND ANALYSIS}

All signal processing was performed off-line using custom scripts in Spike2, and in Matlab using a combination of custom scripts and the program eeglab 6.01b (Delorme and Makeig, 2004). LFP spectra for Figure 5 were made using the multi-taper $\mathrm{fft}$ algorithm PMTM, applied to 1024 samples (205 ms) with a time-bandwidth product of 4 , over $500 \mathrm{~ms}$ windows of data surrounding the inhalation/exhalation (I/E) point of a breath. No significant spectral contribution is observed in the DMSO containing pulses, indicating that observed spectral changes around odor stimulation are not due to artifacts from the stimulation apparatus.

To characterize the response properties in both time and frequency domains we have employed wavelet transformation analysis of individual breaths. Wavelet transformations used the Morlet basis with an initial window size of $446 \mathrm{~ms}$ at 2 cycles, and tapered to 43 cycles. 400 analysis windows were placed within the middle $5.5 \mathrm{~s}$ of each epoch, producing a time bin size of $14 \mathrm{~ms}$. Because analog filtering was not applied before digitization, raw LFP traces contained breath locked theta oscillations with asymmetrical rise and fall times. The rise phase of the theta oscillation had a fundamental frequency in the beta range, so beta power in wavelet spectrograms reflects both beta and theta oscillations.

Spectral power at every frequency band (94 bands from $5 \mathrm{~Hz}$ to $120 \mathrm{~Hz}$ ) was normalized to the average band-power contained in the baseline region (1.5 s after I/E of breath 1). Spectral power above baseline is expressed in decibels $\left[10 \times \log _{10}\right.$ (signal/baseline) $]$. In this way, each odor response was normalized to its own spectral baseline, and the normalized power of repeated odor stimulations for each animal (7-9 per animal) was averaged. Because of this normalization, wavelet powers will not reflect absolute difference in spectral power between strains and conditions, but rather the flux in spectral power over time. Additional normalization to bring the pre-stimulus baseline to zero $\mathrm{dB}$ was done relative to the first three baseline breaths. Compound spectrograms were made by averaging across all animals in a group after the data had been corrected to baseline and normalized. All spectrograms are colored using the same LUT $(-12 \mathrm{~dB}$ to $+12 \mathrm{~dB})$.

\section{PAIRED-PULSE ANALYSIS}

For short ISI ( $<100 \mathrm{~ms}$ ), we observed that both the peak (GC fEPSP) and trough (MC fIPSP) of the second pulse occur on top of a moving baseline created by the first pulse. We assumed the two waveforms overlapped additively, and corrected for this bias by subtracting the value of a single pulse at the time of the event (fEPSP or fIPSP) from 
the second pulse. This bias factor was taken from an average of multiple single stimulations, and the baseline of these single stimulations was set to zero before averaging. Therefore in all paired stimulations with ISI $<100 \mathrm{~ms}$, it is necessary to compare the bias factor to the specific baseline preceding the first stimulus (baseline 1).

The formula for the paired-pulse ratio of two fEPSPs for an ISI less than $100 \mathrm{~ms}$ was:

$$
\frac{\text { peak } 2-(\text { bias - baseline } 1)}{\text { peak } 1 \text { - baseline } 1}
$$

where "peak" is the magnitude of the fEPSP peak, "bias" is the value of the average waveform at the time of the peak, and "baseline" is the value prior to the stimulus artifact. For measurements of fIPSPs, trough 1 was measured from the average trace that was already set to baseline prior to averaging, therefore it was not necessary to compare it to baseline 1 . Thus, the formula for fIPSPs (ISI $<100 \mathrm{~ms}$ ) was:

$$
\frac{\text { trough2 }-(\text { bias }- \text { baseline } 1)}{\text { trough } 1}
$$

For ISI greater than $100 \mathrm{~ms}$, each peak and trough was set to their respective pre-stimulus baseline, so for fEPSPs this was calculated by the following formula:

$$
\frac{\text { peak } 2 \text { - baseline } 2}{\text { peak } 1 \text { - baseline } 1}
$$

fIPSP paired-pulse ratios (ISI>100 ms) were also calculated with formula 3.

\section{ODOR-CUED FEAR CONDITIONING}

All testing occurred during daylight hours and, with few exceptions, at least $2 \mathrm{~h}$ before or after the colony lights cycle. Prior to conditioning the mice were subjected to three 30-min acclimation sessions, each on 3 consecutive days in the fear conditioning chamber during which no CS or US were presented. On day 3 a fear conditioning session consisting of eight CS + US pairing trials with a 4 to 6-min inter-trial-interval was conducted for each mouse. Live observations were made to measure total freezing time for $2 \mathrm{~min}$ after each CS-US trial (Stopwatch+, David A. Brown, http://www. cbn-atl.org/research/stopwatch.shtml).24, 48 and $72 \mathrm{~h}$ after conditioning mice were tested in a different context, the probe chamber, for 20 min: a null stimulus (mineral oil for the odor CS animals) was delivered at $2,4,6$, and $8 \mathrm{~min}$ and the CS was delivered at 10, $12,14,16$, and $18 \mathrm{~min}$. Total freezing was measured throughout the 20-min session. Mice were tested at 6, 21, and 26 weeks after SVZ irradiation or sham treatment; in a parallel and balanced study half of the 26-week cohort was conditioned to an audio tone CS instead of an odor CS. The CS consisted of either a 3 to 5-s odor pulse or a 3 -s audio tone followed immediately by a $2 \mathrm{~s}$ foot shock US.

The fear chamber (interior $30 \mathrm{~cm} \times 24 \mathrm{~cm} \times 25 \mathrm{~cm} \mathrm{H}$ ) has clear acrylic walls providing containment of the shock-grid floor apparatus (Med Associates, model ENV-005-02A) and ventilation sufficient to administer odors as discrete cues. The US consisted of a $0.5 \mathrm{~mA}, 2$-s duration shock provided by a Med Associates ENV-414 shocker scrambler to coincide with administration of odor cues. The probe chamber $(29 \mathrm{~cm} \times 18 \mathrm{~cm} \times 23 \mathrm{~cm} \mathrm{H})$ also has clear acrylic walls and uses the same ceiling fan for exhaust as the fear chamber, but has a smooth acrylic floor instead of the shocking grid floor. Odor is prepared as a $100 \mathrm{mM}$ solution of pentanal (Sigma \#110132) in light mineral oil (Sigma \#330779). $2.5 \mathrm{~mL}$ of odor solution is sealed in a $16 \times 125 \mathrm{~mm}$ culture tube (VWR \#47729-578) with a section of filter paper to act as a wick. A $50 \mathrm{~mL}$ syringe is used to push $50 \mathrm{~mL}$ volume of air through the odor vial (or mineral oil vial for null stimulus trials) and into the testing chamber over 3-5 s. Audio CS was provided via PC speakers to deliver a $2 \mathrm{kHz}$ square wave tone, $3 \mathrm{~s}$ duration, with $75 \pm 5 \mathrm{~dB}$ intensity measured at the floor of the testing chamber.

\section{STATISTICS}

A one-tailed Students $t$-test was used to calculate statistical significance in all comparisons between two distributions. For repeated measures comparisons we used repeated measures ANOVA. All analysis was done in Statview 5.0.1 (SAS, Cary, NC, USA).

\section{RESULTS \\ X-RAY IRRADIATION OF THE MOUSE SUB-VENTRICULAR ZONE PRODUCES A DOSE-DEPENDENT AND LONG-LASTING REDUCTION IN LOCAL CELL PROLIFERATION}

$\mathrm{X}$-ray irradiation is often clinically used to inhibit cancer cell proliferation and brain metastasis. Experimentally it has been successfully applied to ablate adult neurogenesis in the dentate gyrus of the hippocampus (Santarelli et al., 2003), and in the SVZ (Panagiotakos et al., 2007; McGinn et al., 2008; Lazarini et al., 2009). We targeted $\mathrm{x}$-rays to specific regions of the mouse brain by using lead shielding aligned to stereotaxic coordinates. This strategy permitted accurate and reproducible bilateral targeting of the SVZ and a majority of the RMS without exposing the hippocampus or the olfactory epithelium (Figure 1A). Within the window of $x$-ray exposure, the SVZ was largely absent of cell proliferation as measured by 5 -bromodeoxyuridine (BrdU) incorporation measured $24 \mathrm{~h}$ after a single injection (Figure 1B). Targeted irradiation did not alter adult neurogenesis in the dentate gyrus verified by doublecortin (dcx) staining (Figure 1C). The dosage of irradiation was calibrated by changing the duration of exposure on each of 3 days and measuring BrdU incorporation in the SVZ 2 weeks after the last day of irradiation. The most effective dose we tried was calculated to be equivalent to 15 Gy of ionization (Figure 1D, see methods, BrdU + nuclei per SVZ \pm SEM; 0 Gy $550 \pm 38.1 ; 1$ Gy $640 \pm 85.7 ; 5$ Gy $450 \pm 37.8 ; 10$ Gy $136 \pm 10.7 ; 15$ Gy $45 \pm 8.7 ; n=3-9$ per group, significance between $0 \mathrm{~Gy}$ and $15 \mathrm{~Gy}$ and $0 \mathrm{~Gy}$ and $10 \mathrm{~Gy}$, unpaired $t$-test $p<0.001$ ), which is similar to what has been previously reported (Mizumatsu et al., 2003; Santarelli et al., 2003; McGinn et al., 2008). In addition, a significant depression of cell proliferation in the SVZ persisted for over 4 months following exposure (Figure 1E, BrdU + nuclei per 15 Gy SVZ \pm SEM; 3 -days post $x$-ray $7 \pm 2$.4; 7-days post $\mathrm{x}$-ray $45 \pm 8.7 ; 14$-days post $\mathrm{x}$-ray $67 \pm 8.9 ; 28$-days post $\mathrm{x}$-ray $34 \pm 4.0$; 120 -days post $\mathrm{x}$-ray $51 \pm 15.6$; pooled 0 Gy sham controls $525 \pm 18.8$; $n=3-10$ per group, unpaired $t$-test $p<0.001)$.

\section{SURVIVAL OF YOUNG ADULT-BORN NEURONS IN THE OLFACTORY BULB IS UNCHANGED BY REDUCING NEUROGENESIS}

Young adult-born neurons undergo activity-dependent apoptosis during a critical period 2-4 weeks after cell birth (Yamaguchi and Mori, 2005; Mouret et al., 2008). We wanted to measure if SVZ 

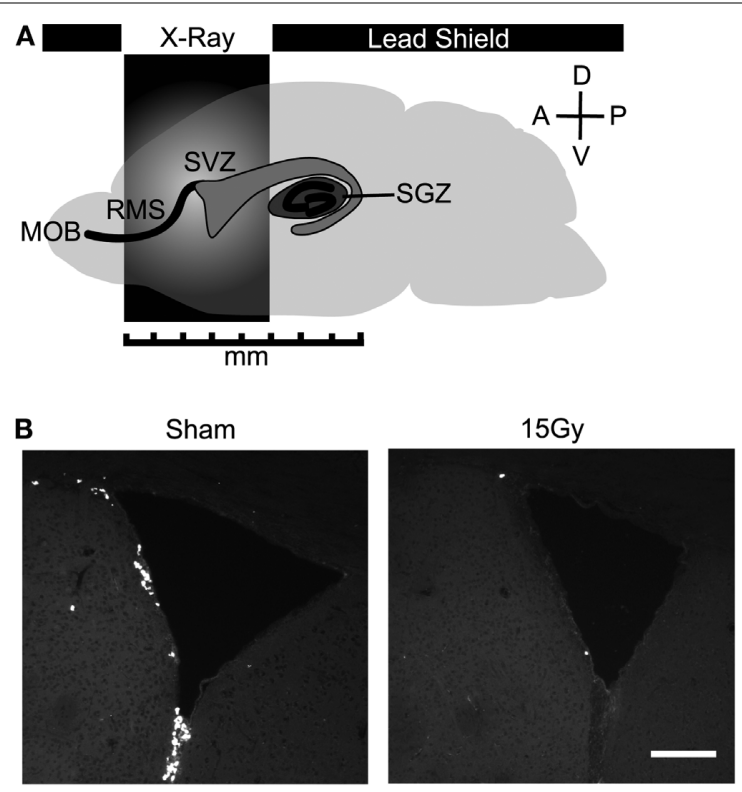

C

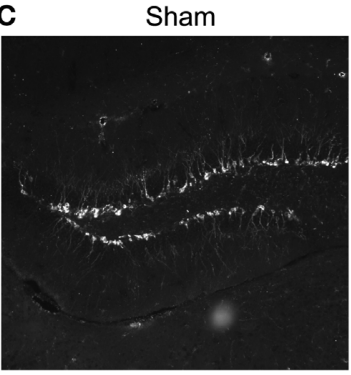

D

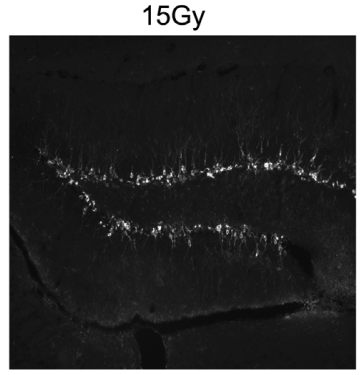

E
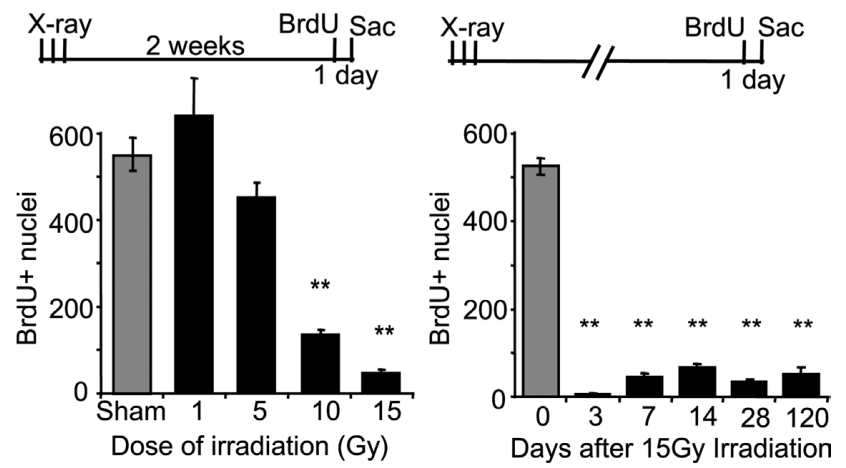

FIGURE 1 | X-ray irradiation of the sub-ventricular zone produces a longlasting reduction in cell proliferation. (A) Schematic showing the placement of the $x$-ray window ( $5 \mathrm{~mm} A-P \times 13 \mathrm{~mm} \mathrm{M}$-L). (B) BrdU positive nuclei along the SVZ were mostly eliminated weeks to months following irradiation. Scale bar $=100 \mu \mathrm{m}$. (C) X-ray targeting did not alter proliferation in the dentate gyrus observed using doublecortin, a marker for immature migrating neuroblasts.

(D) A dose-dependent reduction in SVZ proliferation was produced by controlling the exposure time during the 3-day treatment. Significantly decreased proliferation relative to controls occurred at 10 and 15 Grays, and the maximally effective exposure was calculated to be 15 Grays ( $n=3$ per irradiated group, $n=9$ for controls). (E) A significant depression in SVZ proliferation was observed up to 4 months in irradiated mice compared to agematched controls ( $n=3-6$ for irradiated groups, $n=10$ for pooled controls). **indicates $p<0.001$ compared to non-irradiated control (grey) unpaired $t$-test, error bars indicate SEM. irradiation altered neuronal survival throughout this process. One month after irradiation, a persistent loss of adult-born neurons was qualitatively visualized by a reduction in dcx staining, a label for migrating progenitor cells (Figure 2A). We quantified the loss of neurogenesis to the $\mathrm{OB}$ by acutely injecting the animal with 5-bromo-2'-deoxyuridine (BrdU), and measuring the number of BrdU positive cells that migrated into the GCL. To observe adultborn GCs during their critical period, we counted BrdU positive nuclei 14 and 28 days after BrdU injection, in animals irradiated 3 months prior (Figure 2B). A 15 Gy dose reduced the number of adult born cells of the same age by approximately $96 \% 2$ or 4 weeks after injection (Figure 2C, BrdU nuclei per $\mathrm{mm}^{2} \pm$ SEM 2-weeks post injection: 0 Gy $69.1 \pm 5.1$ (white); 7.5 Gy $29.5 \pm 1.9$ (grey); 15 Gy $2.5 \pm 0.8$ (red); BrdU nuclei per $\mathrm{mm}^{2} \pm$ SEM 4-weeks post injection: 0 Gy $42.0 \pm 7.0$ (white); 7.5 Gy $16.4 \pm 0.7$ (grey); 15 Gy $1.7 \pm 0.2$ (red)). Interestingly, when we compared the loss of BrdU labeled cells between 2 and 4 weeks at full or intermediate doses of irradiation, we found that this loss was not affected by the number of similarly aged cells integrating into the circuit (Percent decrease between 14 and 28 days; 0 Gy 39.2\%; 7.5 Gy 44.4\%; 15 Gy 33.6\%). Thus, to our surprise, we found no compensatory increase in survival of adult born GCs when fewer of them migrate into the OB.

\section{THE NUMBER OF CELLS IN THE OLFACTORY BULB GRANULE CELL LAYER IS REDUCED MONTHS FOLLOWING SVZ IRRADIATION}

Considering that young adult-born GCs continue to die at the normal rate even under conditions of reduced neurogenesis, we next looked to see if GCs of any age showed increased survival. To estimate the total number of cells in the GCL, we combined measurements of density and volume over a period of months after irradiation.

We estimated the volume of the GCL by applying the Cavalieri principle to serial sectioning of the entire tissue (see Materials and Methods). Sagittal sections of the OB of irradiated mice showed a clear depletion of the RMS compared to sham controls (Figure 3A, arrows, compare left to right). The surface area encompassed by the border of the GCL was measured from animals that had received SVZ irradiation 1, 2, 3.5 or 5 months prior. In irradiated animals we found a uniform reduction in the medial to lateral cross-sectional area compared to sham irradiated animals (Figure 3B). This isotropic loss of volume along the medial-lateral axis suggests cell loss in the superficial layers of the GCL (which is present in all sections), rather than collapse of the RMS (present only in the middle sections). Reduced GCL volume in irradiated conditions was significant at all time points compared to sham controls (Figure 3C and Table 1). The time course of this reduction in volume peaked at 2 months; at that point the GCL was $18 \%$ smaller in the X-ray treated group compared to controls. A slight recovery of volume was observed from 2 months to the last measurement at 5 months. This recovery is likely due to a recovery of SVZ proliferation from neurogenic regions at the fringes of the irradiation window, and thus outside the counting window (data not shown).

We next measured the cell density in different regions in the main $\mathrm{OB}$ by combining biased placement of counting windows (Figure 3D) with unbiased counting (the optical dissector, Figure 3E). In this way we could detect changes in known subregions of the GCL with accurate stereological estimation. The 


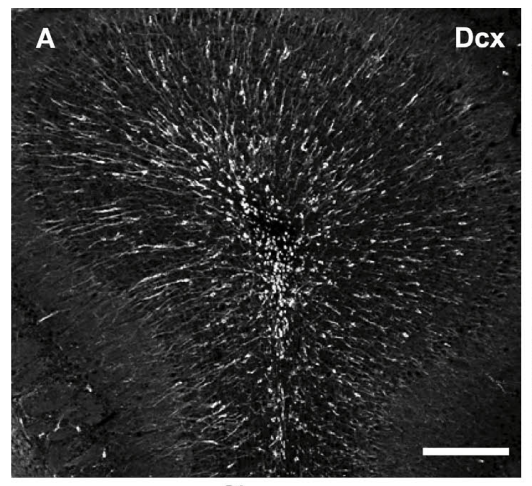

Sham

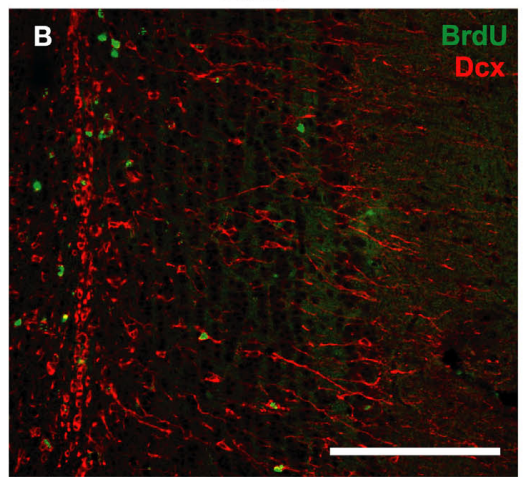

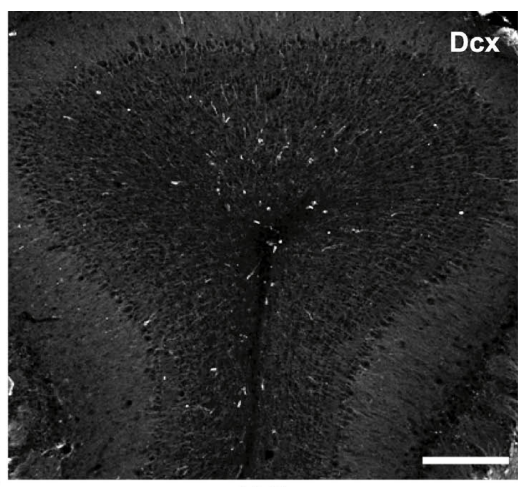

$15 G y$

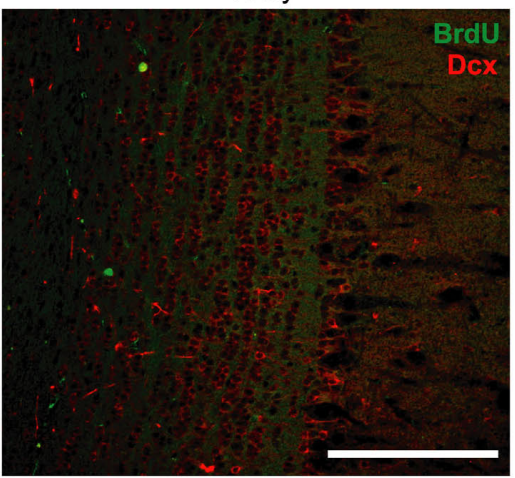

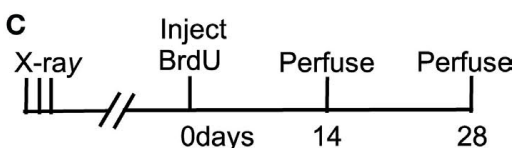

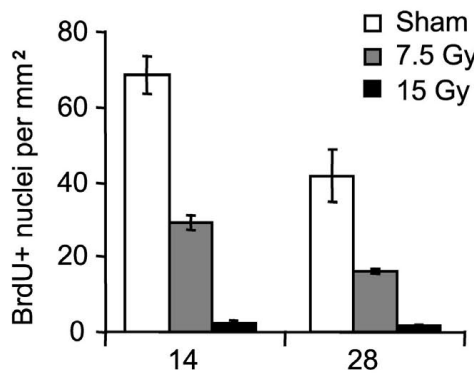

Days post Injection
FIGURE 2 | Reduction in olfactory bulb neurogenesis is not accompanied by compensatory increase in new neuron survival. (A) The magnitude of olfactory bulb neurogenesis is qualitatively reflected by the amount of $d c x(+)$ (Dcx) cells in the granule cell layer. (B) Quantification of cell survival was done by counting BrdU(+) nuclei, shown 4 weeks after irradiation. Scale bars: top $200 \mu \mathrm{m}$, bottom $50 \mu \mathrm{m}$. (C) Animals receiving SVZ irradiation (black) compared to non-irradiated controls (white) have an approximately $96 \%$ reduction in BrdU+ positive nuclei in the olfactory bulb measured 2 and 4 weeks after BrdU injection. Animals receiving a half dose of x-rays (grey) have an approximately $59 \%$ decrease in BrdU+ nuclei compared to non-irradiated controls (white, $n=3-4)$. Between 2 and 4 weeks after cell birth, the number of neurons decreased by approximately $39 \%$. This decrease varied little according to the dose of irradiation (39.2\% at $0 \mathrm{~Gy} ; 44.4 \%$ at $7.5 \mathrm{~Gy} ; 33.6 \%$ at $15 \mathrm{~Gy}$ ). Error bars indicate SEM. density of DAPI-stained nuclei in the GCL was measured in the medial, ventral or dorsal GCL in mice irradiated 1 and 4 months prior. No significant differences were observed between age groups or between anatomical regions (ANOVA with post hoc PLSD, $p>0.05$ ), so data were pooled (Figure 3F). The density of cells in the GCL of irradiated animals $\left(1.102 \pm 36.5\right.$ nuclei $\times 10^{3}$ per $\left.\mathrm{mm}^{3}\right)$ was not significantly different from that of non-irradiated animals $\left(1.128 \pm 43.7\right.$ nuclei $\times 10^{3}$ per $\mathrm{mm}^{3}$, unpaired $t$-test $\left.p>0.05\right)$.

From these measurements of volume and density, we could calculate the change in the total number of cells in the GCL after irradiation. With an average density of cells of 1.1 million per $\mathrm{mm}^{3}$, the reduction in GCL volume indicates that 1 month after irradiation the tissue lost $\sim 368,000$ cells, and 2 months after irradiation it lost $\sim 590,000$ cells. At both time points, this indicates a similar rate of loss $(\sim 12,000$ per day over 1 month; $\sim 10,000$ per day over 2 months).

\section{IRRADIATED MICE HAVE DIMINISHED CONDITIONED RESPONSE IN AN ODOR-CUED FEAR CONDITIONING TASK}

The proliferation and survival of adult born GCs is strongly linked to the animal's environment. We therefore sought to determine if the reduction of granule cell proliferation would produce an observable behavioral effect in a challenging environment.
We developed a model of odor-cued fear conditioning to assess olfactory processing in mice with diminished adult neurogenesis. In our odor-cued fear conditioning paradigm we paired an olfactory conditioned stimulus (CS) with an aversive footshock unconditioned stimulus (US). After one session of conditioning, animals formed a fearful association to the CS exhibited by their freezing behavior. We probed this association in a novel context by measuring the animal's freezing conditioned response (CR) to the $\mathrm{CS}$ in the absence of the US (Figure 4A). Using cued rather than contextual conditioning allows us to assess the $\mathrm{CR}$ as it pertains to the CS specifically, and assess the animal's behavior in the absence of the CS. This is illustrated (Figure 4B) for a $20 \mathrm{~min}$ long probe session with data from all mice pooled into either irradiated (XR) or control (Sham) groups: the CS, in the form of a brief odor pulse, results in a dramatic increase in freezing behavior during the 1-min time bins following CS delivery, the "post-CS minute". During the 10 min prior to CS delivery the odor vehicle, mineral oil, was pulsed into the probe chamber. While the XR and Sham mice did not differ in level of locomotor activity (data not shown) or in the amount of baseline freezing in the probe session (data not shown), it was observed that irradiated mice freeze less in response to the odor CS during the post-CS minute (XR $n=17$ and sham $n=18$; repeated measures ANOVA, $p<0.05$ ) (Figure 4B). 

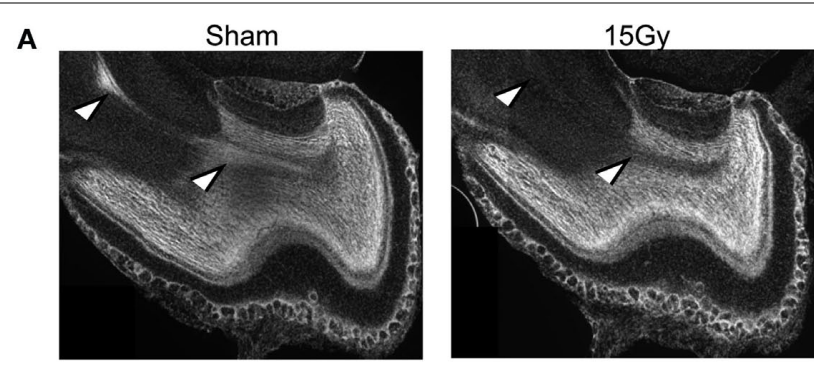

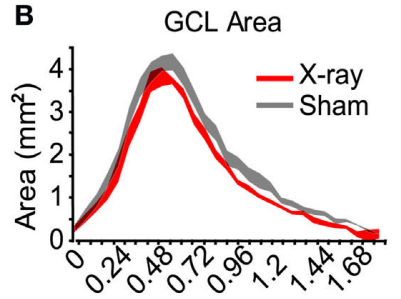

Distance from medial edge $(\mathrm{mm})$

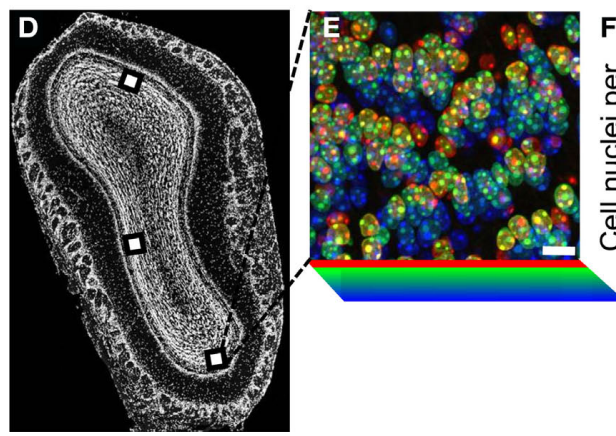

FIGURE 3 |The number of cells in the granule cell layer is reduced following irradiation. (A) Sagittal sections of sham (left) and irradiated tissue (right). Sectioning the entire medial-lateral extent of the olfactory bulb followed by DAPI staining allowed surface area measurement of serial planes along the volume of interest. The rostral migratory stream is indicated by arrows, and is present in sham-treated tissue (left) but nearly absent in irradiated tissue (right). (B) Cross-sectional area of the granule cell layer for data grouped across all time points. (C) The volume encompassed by the edge of the GCL declined in the $x$-rayed condition (solid red line) compared to controls (dotted line) between 1 and 5 months after irradiation (values represent group means, for statistics see Table 1). (D) Example density counting windows placed in an irradiated GCL. (E) Implementation of the optical disector to estimate cell density from highmagnification z-stacks of GCL nuclei false-colored according to z-depth, scale $=10 \mathrm{um}$. (F) Density of granule cell layer from mice receiving sham treatment (grey), and irradiation (red). This data represents the average of multiple anatomical regions, and durations between 1 and 5 months after irradiation. We observed no trend in density over time. All error bars indicate SEM.

As further evidence that this reduction in freezing is related to the odor CS and not a general deficit in freezing behavior, the level of freezing achieved during conditioning did not differ between the groups (Figure 4C). As a control for off-target effects of irradiation on fear and motor circuitry, we used an audio tone instead of an odor as the CS and did not observe any reduction in freezing between irradiated and sham groups (Figure 4D). This indicates that the diminished CR is specific to the odor modality and that the phenotype we see for XR mice is odor dependent. Finally, the reduced freezing is consistent in each of the three probe sessions
Table 1 | Reduction in volume of granule cell containing layers of the olfactory bulb granule cell layer after SVZ irradiation.

\begin{tabular}{llllll}
\hline Condition & Treatment & Volume $\left(\mathbf{m m}^{3}\right)$ & \%change & $\boldsymbol{p}$-value & $\boldsymbol{n}$ \\
\hline \multirow{2}{*}{+1 month } & Sham & $2.935(0.055)$ & & & 3 \\
& XR & $2.600(0.082)$ & -11.41 & $<0.05$ & 3 \\
+2 months & Sham & $2.935(0.122)$ & & & 5 \\
& XR & $2.399(0.032)$ & -18.27 & $<0.05$ & 3 \\
+3.5 months & Sham & $2.359(0.075)$ & & & 4 \\
& XR & $1.967(0.083)$ & -16.61 & $<0.05$ & 3 \\
+5 months & Sham & $2.817(0.112)$ & & & 4 \\
& XR & $2.478(0.051)$ & -12.04 & $<0.05$ & 4
\end{tabular}

Shown is the volume encompassed by the edge of the main olfactory bulb granule cell layer. The difference between the volume of sham and x-ray (XR) treated mice is expressed as the \%change of the $x$-ray condition from the sham condition $\{[(X R / S h a m)-1] \times 100\}$. Significance was evaluated using a one-tailed t-test between Sham and XR mice at each time after treatment.

following odor-cued conditioning (repeated measures ANOVA $p<0.05$; Figure 4E). Importantly, this effect was observed in three cohorts of mice tested 6,21 , and 26 weeks after SVZ irradiation; their data is pooled here (XR $n=17$, sham $n=18)$.

\section{DENDRO-DENDRITIC SYNAPTIC PLASTICITY IN-VIVO IS UNCHANGED IN THE ABSENCE OF YOUNG ADULT-BORN NEURONS, AND AFTER GRANULE CELL LAYER CELL LOSS}

To probe the $\mathrm{OB}$ for electrophysiological changes that may mirror the behavioral phenotype, we first measured the properties of the synapse formed between GCs and mitral/tufted (M/T) cells. Antidromic stimulation of the M/T cell axon bundle in the lateral olfactory tract (LOT) excites this synapse, producing a field potential reflecting glutamatergic granule cell excitation followed by GABAergic M/T cell inhibition (Rall and Shepherd, 1968; Nicoll, 1969; Mori and Takagi, 1978) (Figure 5A). We used the magnitude of the field EPSP (fEPSP) and field IPSP (fEPSP) to reflect aggregate synaptic strength (Figure 5B).

We first sought to determine if, in the absence of young adultborn neurons, the population of GCs showed less excitability. This may arise if young adult-born neurons are intrinsically hyper-excitable, as previously suggested (Belluzzi et al., 2003; Saghatelyan et al., 2005). No significant differences were observed in the stimulus response relationship at any age in either the magnitude of the GC response (fEPSP magnitude; Figure 5C, left, data grouped across all time points, unpaired $t$-test $p>0.05$ ) or in the input-output function of the response (stimulus strength at half-maximal fEPSP; Figure 5C, right, data grouped across all time points, unpaired $t$-test $p>0.05)$.

We next looked at the short-term plasticity of the dendrodendritic synapse at different times after irradiation to probe for changes to pre or post-synaptic function. For this we stimulated the LOT using a paired-pulse paradigm which reveals facilitation of the GC fEPSP (Figures 5D-F, left) and depression of the reciprocally evoked mitral and tufted cell fIPSP (Figures 5D-F right) at short inter-stimulus intervals (ISI). Between 1 and 5 months after irradiation, no systematic changes were observed in the plasticity profiles of this synapse, nor were differences observed within any individual time point (repeated measures ANOVA $p>0.05$ ). 


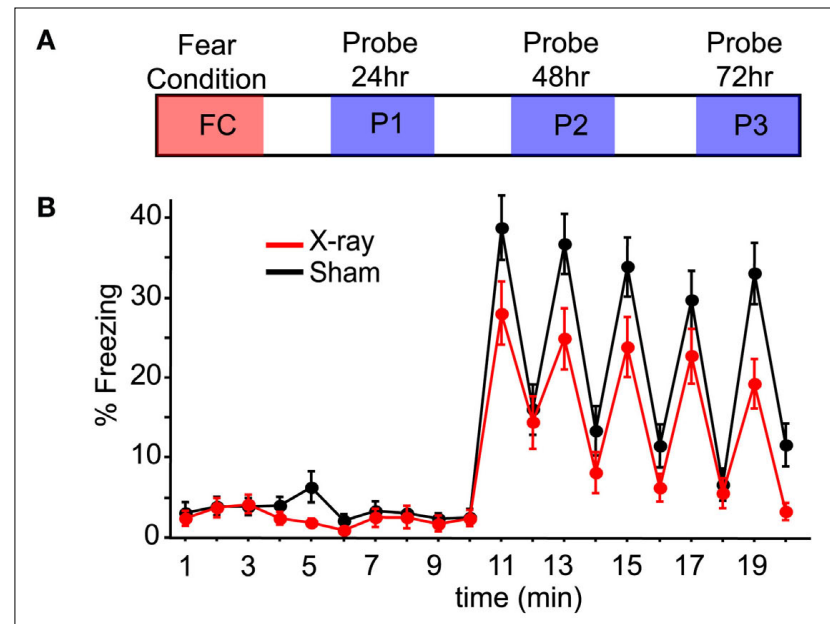

C

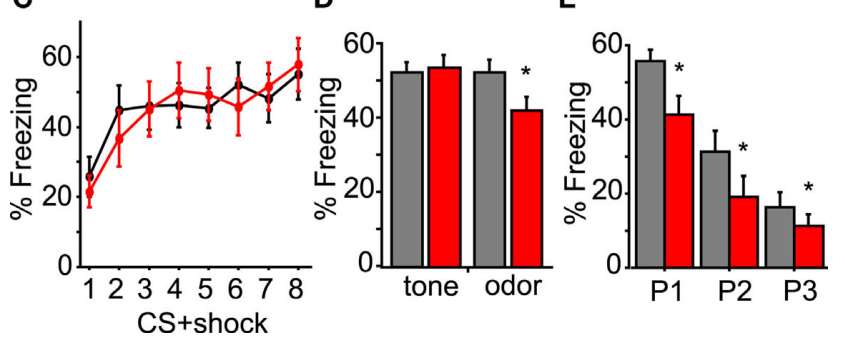

FIGURE 4 | Irradiated mice show normal acquisition of fear conditioning, but display less odor-cued freezing. (A) An illustration of the timeline of testing: Fear Conditioning consists of a 40-min session that includes 8 $\mathrm{CS}+$ shock pairings with a variable inter-trial interval of 4-6 min. At 24, 48, and $72 \mathrm{~h}$ after conditioning a 20-min probe session is conducted in a novel context. (B) Time course of a probe of odor-cued conditioned freezing response. Plotted is the mean amount of freezing as a percentage of each minute in the 20-min probe; all three probe sessions are included in the calculation of mean percent freezing. All mice conditioned to odor are grouped: $X R n=17$ (red) and sham $n=18$ (black). The increase in freezing in the minute immediately following CS (minutes 11, 13, 15, 17, and 19) is the "post-CS minute" that is used in subsequent analysis of freezing. (C) Acquisition of freezing response during conditioning. The average percent freezing is plotted for $X R(n=17)$ and sham ( $n=18$ ) mice; here the percent freezing is during the 2 min immediately following each CS + shock pairing. Only mice conditioned to odor are represented; mice conditioned to an audio tone displayed a similar pattern for acquisition of freezing behavior (data not shown). (D) Members of a cohort of mice tested 26-weeks post-irradiation were conditioned to a $2 \mathrm{kHz}$ audio tone rather than odor. In this graph mean percent freezing from all three probe sessions for the +26-week cohort is plotted; the reduced freezing displayed by the XR group (factorial ANOVA $n=10, p<0.05$ ) in response to a conditioned odor was not observed for mice conditioned to an audio tone $(n=10)$. (E) The average percent freezing for the "post-CS minute" during each of the three probe sessions is plotted. XR mice $(n=17)$ freeze less than Sham mice $(n=18)$; repeated measures ANOVA $p<0.05$. In factorial ANOVA P1 and P2 are $p<0.05$, and for $\mathrm{P} 3 p=0.0627$. All error bars represent SEM.

\section{LOCAL FIELD POTENTIAL RESPONSES TO ODORANT STIMULATION REMAIN UNPERTURBED MONTHS FOLLOWING IRRADIATION}

Having observed no changes to the dendro-dendritic synapse in a bulb largely absent of new adult-born neurons, we reasoned that functional changes may only emerge if odors are used as stimuli. To gain a system-wide perspective of how odors are processed in the $\mathrm{OB}$, we measured local field potential (LFP) oscillations which, when measured in the deep GCL, largely reflect aggregate coherent synaptic activity in the external plexiform layer (Rall and Shepherd, 1968). In addition, distinct frequency bands of LFP oscillations can be tied to specific mechanisms; for instance, gamma oscillations are produced by the mitral to granule cell dendro-dendritic synapse (Schoppa, 2006; Lagier et al., 2007; Kay et al., 2009).

In freely breathing anesthetized mice, we recorded the LFP oscillations that emerge around an odor stimulus. During normal respiration we observed prominent slow oscillations locked to the breathing rate (Figure 6B), with embedded higher frequency oscillations emerging and decaying within the course of a breath. We measured the relationship between odor concentration and evoked LFP power in beta frequency oscillations (Figure 6C, 10-40 Hz), gamma frequency oscillations (Figure 6D, 40-70 Hz), and high gamma oscillations (Figure 6E, 70-100 Hz) from mice 1 and 2 months after irradiation. No significant differences emerged at either time point, or when the data were pooled (Figures 6C-E, unpaired $t$-test, $p>0.05$ ).

Neural activity in the $\mathrm{OB}$ is tightly constrained by the breathing cycle (Bathellier et al., 2008; Wesson et al., 2008), so finer spectral analysis was needed to extract how LFP frequencies emerge and decay within a breath. We turned to wavelet techniques that can estimate the power of LFP oscillations with high temporal resolution (see Materials and Methods). This data can be visualized by frequency-time spectrograms which we centered on the time when the mouse transitions from inhalation to exhalation (Figure 6F). Analysis of this data at different times after irradiation failed to reveal any fine temporal disruptions in the emergence of neural activity within a breath, illustrated by the profile of oscillatory power in the gamma (Figure 6G, top) and beta bands (Figure 6G, bottom) measured 1-2 months after irradiation (repeated measures ANOVA, $p>0.05)$.

\section{DISCUSSION}

Adult neurogenesis in the OB has been known and investigated for more than two decades, but a clear function for this robust cellular production, nearly unique in the vertebrate brain, has been elusive. Here we provide new evidence for the function of adult neurogenesis in the OB granule cell population. We produced a 20 -fold reduction in the number of incoming adult-born neuroblasts, and observed a behavioral deficit in odor-cued fear conditioning. However, in spite of a reproducible behavioral effect, we were unable to identify any corresponding electrophysiological change measured from the altered GCL. Surprisingly, a variety of electrophysiological tests showed normal GCL circuit function as the standing population of old GCs declined in number by up to $18 \%$ compared to the non-irradiated condition. This suggests robust functional homeostasis in a circuit with a declining proportion of interneurons.

A further unexpected finding was that, in parallel with the slow attrition of the GCL population, survival of young adult-born GCs was unchanged after SVZ irradiation. Specifically, survival of young neurons during their "critical period" between 2 and 4 weeks of cell age (Petreanu and Alvarez-Buylla, 2002; Yamaguchi and Mori, 2005) remained constant regardless of the level of SVZ proliferation. As a result, survival of young adult-born GCs in this circuit is likely not determined by competition for scarce trophic resources contrary to hippocampal adult neurogenesis (Sairanen et al., 2005) and classical 

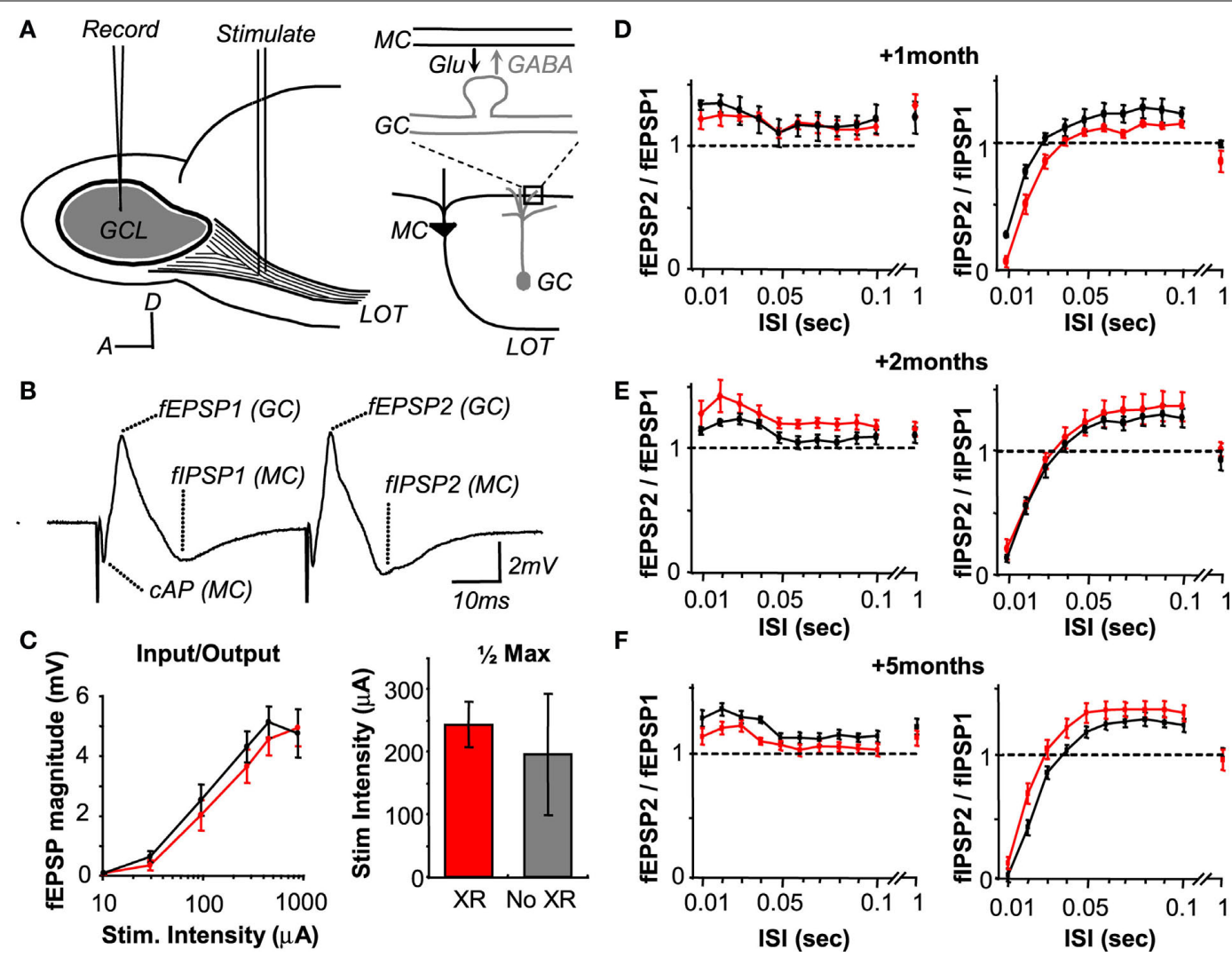

FIGURE 5 | Loss of neurogenesis does not change the activation and plasticity of the dendro-dendritic synapse in-vivo. (A) Schematic of the stimulation paradigm, with a diagram of the dendro-dendritic circuit between mitral cells (MC) and granule cells (GC). (B) The waveform following stimulation has three distinct phases corresponding to the mitral cell compound action potential (CAP), the field EPSP (fEPSP) originating in the granule cells, and the reciprocal field IPSP (fIPSP) in mitral cells.

neurotrophic regulation of survival in the peripheral nervous system (Levi-Montalcini, 1987). Thus, the extent of neurogenesis from the SVZ had no effect on either the physiology or the dynamics of neuronal turnover within this tissue. Nevertheless, the robust behavioral phenotype in irradiated mice is the first evidence that adult SVZ neurogenesis has functional relevance to an extensive behavioral system beyond the OB.

The fear conditioning paradigm probes the interaction between olfactory processing and the amygdala (Sah et al., 2003). In rats, lesions of the amygdala are known to disrupt the association of CS and US regardless of stimulus modality or response measure (Blanchard and Blanchard, 1972). The basic mechanism involves association within the amygdala of the US, a foot shock, with a CS, here an odor pulse that is paired with foot shock. Projections from the amygdala control the fear response and target sensory areas including the olfactory cortex which, along with the $\mathrm{OB}$, return projections to amygdalar nuclei (Scalia and Winans, 1975; Shepherd, 1998).

We observed a diminished fear response of SVZ irradiated mice to a fear-conditioned odor, despite successful formation of the association. This phenotype did not appear when an auditory stimulus was used, and it did not reflect any disruption in their

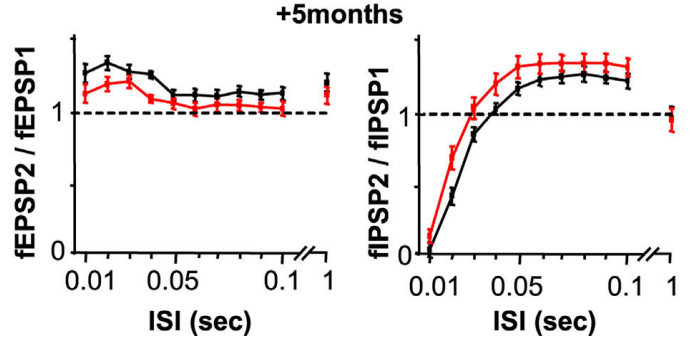

(C) Input-output function relating intensity of $100 \mu \mathrm{s}$ LOT stimulation to fEPSP magnitude from all animals. The current that evokes a half-maximal response is depicted on the right. (D-F) Paired-pulse stimulation of the synapse at maximal intensity, and over a range of inter-stimulus intervals (ISI) from $10 \mathrm{~ms}$ to $1 \mathrm{~s}$. This paradigm reveals minor facilitation (values $>1$ ) in the granule cell fEPSP (left) and large depression (values $<1$ ) in the mitral cell fIPSP (right).

ability to exhibit fear during the formation of the association. In addition, recent work has shown that SVZ irradiated mice posses unaltered detection thresholds and a normal capacity for fine odor discrimination, indicating that our phenotype was not the result of disrupted sensitivity to the conditioned stimulus (Lazarini et al., 2009). Intriguingly, the aforementioned study also found disrupted long-term recall of a positive-valence association, which, when taken together with our observed phenotype, may generally implicate young adult-born GCs in the recall or maintenance of both positive and negative valence associations.

This phenotype could result from the acute loss of young adult born neurons in the MOB, or after months due to the slow decline in interneuron population size. As we are unable to rescue adult SVZ proliferation in the $\mathrm{x}$-ray model we cannot directly test if an acute loss of adult born cells alone is sufficient to generate this phenotype. Nonetheless, we observed this behavioral effect at three time points after irradiation, each with a different amount of GCL cell loss, suggesting our behavioral phenotype is the result of an acute loss of young adult born neurons. In addition, the phenotype was consistently observed 6-26 weeks after irradiation, ruling out the off-target effects of cranial irradiation such as inflammation (Monje et al., 2002). 


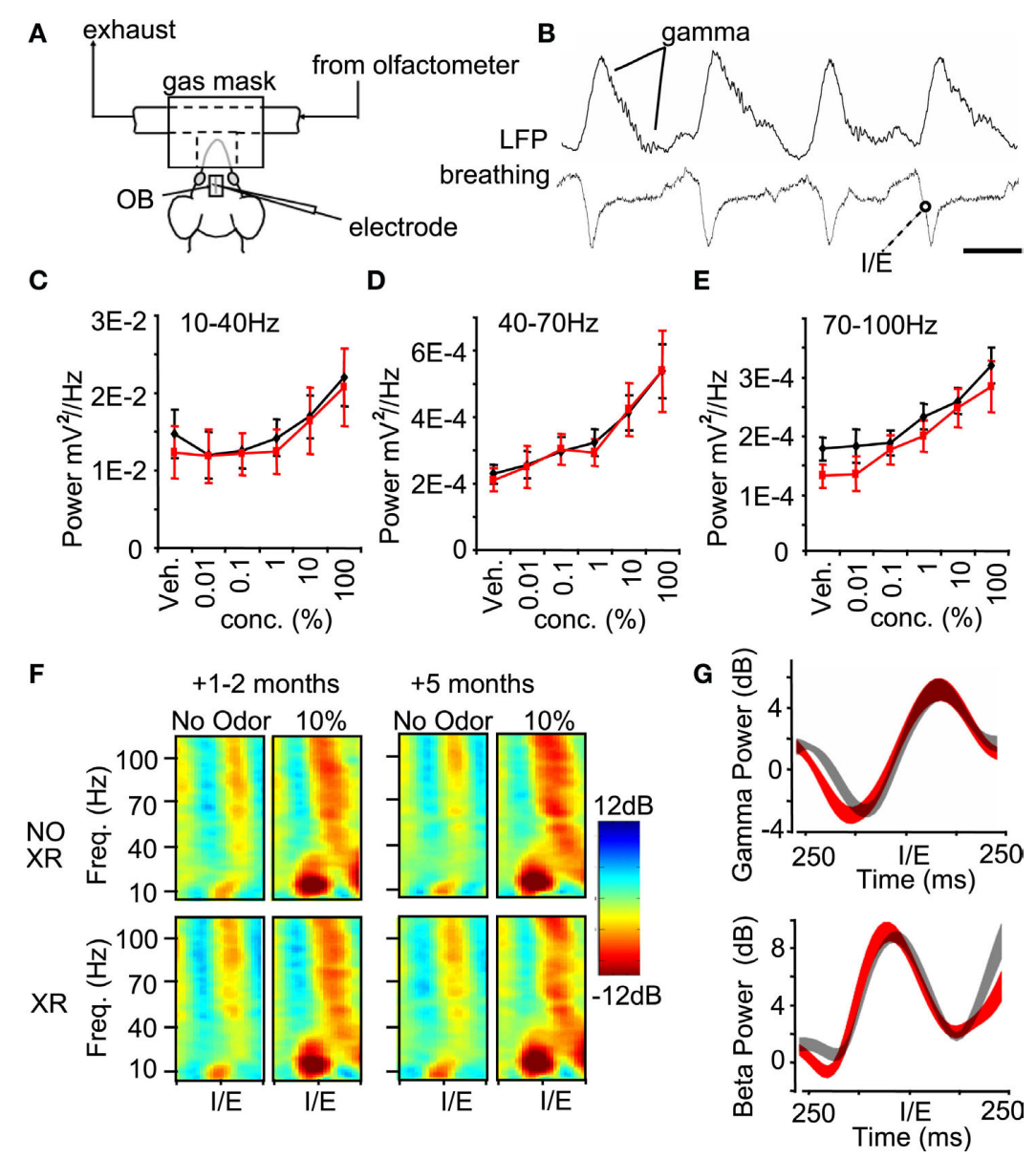

FIGURE 6 | Oscillatory dynamics in the olfactory bulb are unchanged months following a reduction of neurogenesis. (A) In vivo recording paradigm, identical to that in Figure 4, highlighting the odor delivery apparatus. (B) Local field potential (LFP) traces (top) are recorded alongside the breathing rate (bottom). Gamma frequency $(40-100 \mathrm{~Hz})$ oscillations are highlighted emerging after a breath. The transition between inhalation and exhalation (I/E) is used as the trigger for all analyses and odor delivery (Scale $=250 \mathrm{~ms}$ ). Spectral density of beta (C), gamma (D) and high gamma (E) LFP oscillations measured after stimulation with increasing concentrations of the odor Amyl Acetate with

Our observed behavioral phenotype may result from altered centrifugal modulation of the OB. Specifically, odor-cued fear conditioning may use the centrifugal systems that feed back onto young adult-born GCs. Anatomically, the medial amygdalar nuclei connect to the OB through the olfactory peduncles and piriform cortex (Shepherd, 1998). Recent work suggests that the proximal dendrites of GCs, which receive inputs from piriform cortex among other regions, function as the gatekeeper of information from different brain modalities into the OB (Balu et al., 2007), and exhibit unique plasticity in young adult-born neurons (Nissant et al., 2009). Our behavioral phenotype was also similar to changes in contextual freezing behavior observed after ablating hippocampal adult neurogenesis (Saxe et al., 2006). As suggested in the dentate gyrus, young adult-born olfactory GCs may be particularly influenced by high-valence stimuli or states of arousal, potentially through their muscarinic, cholinergic, (Castillo et al., 1999; Linster and Cleland, 2002) or noradrenergic synaptic inputs (Doucette et al., 2007). In vehicle DMSO. Recordings were performed 1-2 months after sham (black) or irradiation (red) treatment ( $n=9-10$ per group). (F) Frequency-time spectrogram showing fine temporal analysis of oscillatory power over 500 ms centered around a breath. Each spectrogram represents the average of its dataset ( $n=9-10$ for all groups). (G) Extracting beta $(10-40 \mathrm{~Hz})$ and gamma $(40-70 \mathrm{~Hz})$ band-power from the 1-2 month spectrograms shows how stimulation with 10\% Amyl Acetate produces a distinct profile of power within the breathing cycle, but without significant differences between groups. The average \pm SEM is depicted for sham (grey) and irradiated (red) animals.

high-valence olfactory environments these pathways may act on GCs in concert with proximal synaptic inputs from the cortex, recruiting specifically young adult born GCs into new patterns of functional connectivity.

In search of physical loci for the behavioral phenotype, we made measurements that reflect general olfactory processing across a wide region of the $\mathrm{OB}$. While these tests are necessarily an incomplete characterization of $\mathrm{OB}$ function, they nonetheless reflect two critical aspects of granule cell function: the generation of oscillatory potentials, and the dendro-dendritic inhibition of mitral and tufted cells. In a previous report, locally generated field potentials in the OB were sensitive to physiological correlates of olfactory behavior (Le Pichon et al., 2009). Specifically, odor-evoked LFP measurements assay the aggregate current evoked during the processing of a stimulus and thus reflect the magnitude and synchronization of neural activity in a local volume. With their temporal and spectral complexity these oscillations are informationally rich, yet, using the 
most sensitive techniques available we could find no alteration in spectral-temporal patterning in the GCL. With paired-pulse measurements, we monitored short-term synaptic plasticity specifically among the population of dendro-dendritic synapses. This synapse is the only output of the granule cell, and in a previous report was the locus for homeostatic compensation for olfactory deprivation (Saghatelyan et al., 2005). We found no evidence of altered shortterm plasticity, or a change of input gain to either the M/T or GC side of the dendro-dendritc synapse.

In characterizing the $\mathrm{x}$-ray lesion, we found that the survival of young adult-born GCs is uncoupled from the quantity of cells of the same age integrating into the circuit. This indicates that the survival of a neuroblast within its critical period is not regulated by competition with neurons of the same age. Instead, as is well established from the literature, neuroblast survival is sensitive to efferent activity (Petreanu and Alvarez-Buylla, 2002; Rochefort et al., 2002; Winner et al., 2002; Yamaguchi and Mori, 2005; Mandairon et al., 2006) and afferent activity (Alonso et al., 2006; Mouret et al., 2008). This leads to a quandary regarding the maintenance of the GCL: how does the tissue adjust to variations in the amount of SVZ neurogenesis? These variations may occur frequently and unpredictably. SVZ proliferation is modulated by many factors including pregnancy (Shingo et al., 2003), ischemia (Zhang et al., 2004) seizures (Parent et al., 2002) and a multitude of trophic factors such as EGF (Kuhn et al., 1997) and sonic hedgehog (Machold et al., 2003). In the absence of changes in the olfactory environment, from our data we would predict that downward fluctuations in SVZ proliferation deprive the GCL of young adult-born neurons, and upward fluctuations produce over-abundance.

The innate plasticity of GCs may be one way the GCL adapts to fluctuations in neurogenesis (Bonfanti and Theodosis, 2009). Adult born GCs, but not post-natal born GCs, are uniquely capable of long-term synaptic plasticity (Nissant et al., 2009). Furthermore, adult born GCs, but not post-natal born GCs, display increased excitability to compensate for decreased spine density following olfactory deprivation (Saghatelyan et al., 2005). Similar homeostatic mechanisms may emerge after changes in SVZ proliferation to adjust the functional properties of the GCL. These as yet unidentified mechanisms would constitute an important link between SVZ neurogenesis and OB physiology.

While this study did not address the properties of the two other main categories of SVZ-derived adult-born neurons, peri-glomerular cells, and the GCs of the accessory $\mathrm{OB}$, it is quite possible that neurogenesis among these populations has a unique functional purpose. We also cannot rule out the possibility that the behavioral observations stem from changes to these circuits. Interestingly, the

\section{REFERENCES}

Alonso, M., Viollet, C., Gabellec, M. M., Meas-Yedid, V., Olivo-Marin, J.C., and Lledo, P.M. (2006). Olfactory discrimination learning increases the survival of adult-born neurons in the olfactory bulb. J. Neurosci. 26, 10508-10513.

Alvarez-Buylla, A., and Garcia-Verdugo, J. M. (2002). Neurogenesis in adult subventricular zone. J. Neurosci. 22, 629-634.
Balu, R., Pressler, R. T., and Strowbridge, B. W. (2007). Multiple modes of synaptic excitation of olfactory bulb granule cells. J. Neurosci. 27, 5621-5632.

Bathellier, B., Buhl, D. L., Accolla, R., and Carleton,A. (2008). Dynamic ensemble odor coding in the mammalian olfactory bulb: sensory information at different timescales. Neuron 57, 586-598.

Bayer, S. A., Yackel, J. W., and Puri, P. S. (1982). Neurons in the rat dentate

percentage of adult-born neurons in the GCL remains constant at approximately $10 \%$, while the glomerular layer undergoes a net addition of adult-born neurons over time (Ninkovic et al., 2007), similar to neuronal addition in the hippocampus (Bayer et al., 1982), suggesting a unique role for adult neurogenesis in the periglomerular circuit over the life of the animal.

Further defining the functional significance of adult neurogenesis will require sophisticated genetic dissection in concert with multiple behavioral and physiological tests. Neurogenesis in specific subpopulations, such as those defined by protein expression (e.g Pax6), pharmacological profile (e.g adrenergic receptor subtypes), or morphology (e.g inter-glomerular vs. intra-glomerular) may be regulated according to independent mechanisms, and may subserve specific and independent circuit functions. A challenge for the future will be to uncover the range of functions imparted by adult neurogenesis onto these diverse microcircuits. This knowledge is crucial for a program in which these adult born neurons can be of value in other brain regions that have been affected by damage, disease or aging.

\section{ACKNOWLEDGMENTS}

The authors thank members of the Hen laboratory at Columbia University for their assistance and feedback throughout this project, especially Dr. Michael Saxe for help with x-ray experimental design, and Bijan Silari and Pia O'Neill for help with behavioral experiments. Pia Kelsey O'Neil should also be thanked for help with behavior (she did the locomotor observations that were not shown). We also thank Dr. Darcy Kelley of Columbia University and members of the Firestein lab for helpful comments on the manuscript, and Dr. Joshua Gordon of Columbia University for valuable discussions on the electrophysiology data.

\section{AUTHOR CONTRIBUTIONS}

Matthew T. Valley, Tanner R. Mullen and Stuart Firestein wrote the paper. Matthew T. Valley designed and analyzed, and Matthew T. Valley and Lucy C. Schultz conducted histology experiments. Matthew T. Valley and Botir T. Sagdullaev designed, and Matthew T. Valley conducted and analyzed electrophysiology experiments. Tanner R. Mullen designed, conducted, and analyzed behavior experiments.

\section{FUNDING}

The funders had no role in study design, data collection and analysis, decision to publish, or preparation of the manuscript. This work was supported by grant 1R01DC009239-01A1 from the National Institute on Deafness and Other Communication Disorders (Stuart Firestein, Matthew T. Valley, Tanner R. Mullen, Lucy C. Schultz, Botir T. Sagdullaev).

gyrus granular layer substantially increase during juvenile and adult life. Science 216, 890-892.

Belluzzi, O., Benedusi, M., Ackman, J., and LoTurco, J. J. (2003). Electrophysiological differentiation of new neurons in the olfactory bulb. J. Neurosci. 23, 10411-10418.

Blanchard, D. C., and Blanchard, R. J. (1972). Innate and conditioned reactions to threat in rats with amygdaloid lesions. J. Comp. Physiol. Psychol. 81, 281-290.

Bonfanti, L., and Peretto, P. (2007). Radial glial origin of the adult neural stem cells in the subventricular zone. Prog. Neurobiol. 83, 24-36.

Bonfanti, L., and Theodosis, D. T. (2009). Polysialic acid and activity-dependent synapse remodeling. Cell Adh. Migr. 3. Carleton, A., Petreanu, L. T., Lansford, R., Alvarez-Buylla, A., and Lledo, P. M. 
(2003). Becoming a new neuron in the adult olfactory bulb. Nat. Neurosci. 6, 507-518.

Castillo, P. E., Carleton, A., Vincent, J. D., and Lledo, P. M. (1999). Multiple and opposing roles of cholinergic transmission in the main olfactory bulb. J. Neurosci. 19, 9180-9191.

Cecchi, G. A., Petreanu, L. T., AlvarezBuylla,A., and Magnasco, M.O.(2001). Unsupervised learning and adaptation in a model of adult neurogenesis. J. Comput. Neurosci. 11, 175-182.

Delorme, A., and Makeig, S. (2004). EEGLAB: an open source toolbox for analysis of single-trial EEG dynamics including independent component analysis. J. Neurosci. Methods 134, 9-21.

Doucette, W., Milder, J., and Restrepo, D. (2007). Adrenergic modulation of olfactory bulb circuitry affects odor discrimination. Learn. Mem. 14, 539-547.

Gao, Y., and Strowbridge, B. W. (2009). Long-term plasticity of excitatory inputs to granule cells in the rat olfactory bulb. Nat. Neurosci. 12, 731-733.

Hubel, D. H. (1957). Tungsten Microelectrode for Recording from Single Units. Science 125, 549-550.

Imayoshi, I., Sakamoto, M., Ohtsuka, T., Takao, K., Miyakawa, T., Yamaguchi, M., Mori, K., Ikeda, T., Itohara, S., and Kageyama, R. (2008). Roles of continuous neurogenesis in the structural and functional integrity of the adult forebrain. Nat. Neurosci. 11, 1153-1161.

Jahr, C. E., and Nicoll, R. A. (1980). Dendrodendritic inhibition: demonstration with intracellular recording. Science 207, 1473-1475.

Kay, L. M., Beshel, J., Brea, J., Martin, C., Rojas-Libano, D., and Kopell, N. (2009). Olfactory oscillations: the what, how and what for. Trends Neurosci. 32, 207-214.

Kelsch, W., Lin, C. W., and Lois, C. (2008). Sequential development of synapses in dendritic domains during adult neurogenesis. Proc. Natl. Acad. Sci. U.S.A. 105, 16803-16808.

Kuhn, H. G., Winkler, J., Kempermann, G., Thal, L. J., and Gage, F. H. (1997). Epidermal growth factor and fibroblast growth factor- 2 have different effects on neural progenitors in the adult rat brain. J. Neurosci. 17, 5820-5829.

Lagier, S., Panzanelli, P., Russo, R. E., Nissant, A., Bathellier, B., SassoePognetto, M., Fritschy, J.M., and Lledo, P. M. (2007). GABAergic inhibition at dendrodendritic synapses tunes gamma oscillations in the olfactory bulb. Proc. Natl. Acad. Sci. U.S.A. 104, 7259-7264.
Lazarini, F., Mouthon, M. A., Gheusi, G., de Chaumont, F., Olivo-Marin, J. C., Lamarque, S., Abrous, D. N., Boussin, F. D., and Lledo, P. M. (2009). Cellular and behavioral effects of cranial irradiation of the subventricular zone in adult mice. PLoS ONE 4, e7017. doi:10.1371/journal.pone.0007017.

LePichon,C.E., Valley,M.T.,Polymenidou, M., Chesler, A. T., Sagdullaev, B. T., Aguzzi, A., and Firestein, S. (2009). Olfactory behavior and physiology are disrupted in prion protein knockout mice. Nat. Neurosci. 12, 60-69.

Lemasson, M., Saghatelyan, A., OlivoMarin, J. C., and Lledo, P. M. (2005). Neonatal and adult neurogenesis provide two distinct populations of newborn neurons to the mouse olfactory bulb. J. Neurosci. 25, 6816-6825.

Levi-Montalcini, R. (1987). The nerve growth factor 35 years later. Science 237, 1154-1162.

Linster, C., and Cleland, T. A. (2002). Cholinergic modulation of sensory representations in the olfactory bulb. Neural. Netw. 15, 709-717.

Lledo, P.M., Alonso, M., and Grubb, M. S. (2006). Adult neurogenesis and functional plasticity in neuronal circuits. Nat. Rev. Neurosci. 7, 179-193.

Lorig, T. S., Elmes, D. G., Zald, D. H., and Pardo, J. V. (1999). A computercontrolled olfactometer for fMRI and electrophysiological studies of olfaction. Behav. Res. Methods Instrum. Comput. 31, 370-375.

Machold, R., Hayashi, S., Rutlin, M., Muzumdar, M. D., Nery, S., Corbin, J. G., Gritli-Linde, A., Dellovade, T., Porter, J. A., Rubin, L. L., Dudek, H., McMahon, A. P., and Fishell, G. (2003). Sonic hedgehog is required for progenitor cell maintenance in telencephalic stem cell niches. Neuron 39, 937-950.

Mak, G. K., Enwere, E. K., Gregg, C., Pakarainen, T., Poutanen, M., Huhtaniemi, I., and Weiss, S. (2007). Male pheromone-stimulated neurogenesis in the adult female brain: possible role in mating behavior. Nat. Neurosci. 10, 1003-1011.

Mandairon, N., Sacquet, J., Garcia, S., Ravel, N., Jourdan, F., and Didier, A. (2006). Neurogenic correlates of an olfactory discrimination task in the adult olfactory bulb. Eur. J. Neurosci. 24, 3578-3588.

McGinn, M. J., Sun, D., and Colello, R. J. (2008). Utilizing X-irradiation to selectively eliminate neural stem/progenitor cells from neurogenic regions of the mammalian brain. J. Neurosci. Methods 170, 9-15.

Mizumatsu, S., Monje, M. L., Morhardt, D. R., Rola, R., Palmer, T. D., and Fike, J. R. (2003). Extreme sensitivity of adult neurogenesis to low doses of X-irradiation. Cancer Res. 63, 4021-4027.

Monje, M. L., Mizumatsu, S., Fike, J. R., and Palmer, T. D. (2002). Irradiation induces neural precursor-cell dysfunction. Nat. Med. 8, 955-962.

Mori, K., and Takagi, S. F. (1978). An intracellular study of dendrodendritic inhibitory synapses on mitral cells in the rabbit olfactory bulb. J. Physiol. (Lond.) 279, 569-588.

Mouret, A., Gheusi, G., Gabellec, M. M., de Chaumont, F., Olivo-Marin, J. C., and Lledo, P. M. (2008). Learning and survival of newly generated neurons: when time matters. J. Neurosci. 28, 11511-11516.

Nicoll, R. A. (1969). Inhibitory mechanisms in the rabbit olfactory bulb: dendrodendritic mechanisms. Brain Res. 14, 157-172.

Ninkovic, J., Mori, T., and Gotz, M. (2007). Distinct modes of neuron addition in adult mouse neurogenesis. J. Neurosci. 27, 10906-10911.

Nissant, A., Bardy, C., Katagiri, H., Murray, K., and Lledo, P. M. (2009). Adult neurogenesis promotes synaptic plasticity in the olfactory bulb. Nat. Neurosci. 12, 728-730.

Panagiotakos, G., Alshamy, G., Chan, B., Abrams, R., Greenberg, E., Saxena, A., Bradbury, M., Edgar, M., Gutin, P., and Tabar, V. (2007). Long-term impact of radiation on the stem cell and oligodendrocyte precursors in the brain.PLoS ONE 2, e588. doi:10.1371/ journal.pone.0000588.

Parent, J. M., Valentin, V. V., and Lowenstein, D. H. (2002). Prolonged seizures increase proliferating neuroblasts in the adult rat subventricular zone-olfactory bulb pathway. $J$. Neurosci. 22, 3174-3188.

Petreanu, L., and Alvarez-Buylla,A. (2002). Maturation and death of adult-born olfactory bulb granule neurons: role of olfaction. J. Neurosci. 22, 6106-6113.

Rall, W., and Shepherd, G. M. (1968). Theoretical reconstruction of field potentials and dendrodendritic synaptic interactions in olfactory bulb. J. Neurophysiol. 31, 884-915.

Rochefort, C., Gheusi, G., Vincent, J. D., and Lledo, P. M. (2002). Enriched odor exposure increases the number of newborn neurons in the adult olfactory bulb and improves odor memory. J. Neurosci. 22, 2679-2689.

Saghatelyan, A., Roux, P., Migliore, M., Rochefort, C., Desmaisons, D., Charneau, P., Shepherd, G. M., and Lledo, P. M. (2005). Activity-dependent adjustments of the inhibitory network in the olfactory bulb following early postnatal deprivation. Neuron 46, 103-116.
Sah, P., Faber, E. S., Lopez De Armentia, M., and Power, J. (2003). The amygdaloid complex: anatomy and physiology. Physiol. Rev. 83, 803-834.

Sairanen, M., Lucas, G., Ernfors, P., Castren, M., and Castren, E. (2005). Brain-derived neurotrophic factor and antidepressant drugs have different but coordinated effects on neuronal turnover, proliferation, and survival in the adult dentate gyrus. J. Neurosci. 25, 1089-1094.

Santarelli, L., Saxe, M., Gross, C., Surget, A., Battaglia, F., Dulawa, S., Weisstaub, N., Lee, J., Duman, R., Arancio, O., Belzung, C., and Hen, R. (2003). Requirement of hippocampal neurogenesis for the behavioral effects of antidepressants. Science 301, 805-809.

Saxe, M. D., Battaglia, F., Wang, J. W., Malleret, G., David, D. J., Monckton, J. E., Garcia, A. D., Sofroniew, M. V., Kandel, E. R., Santarelli, L., Hen, R., and Drew, M. R. (2006). Ablation of hippocampal neurogenesis impairs contextual fear conditioning and synaptic plasticity in the dentate gyrus. Proc. Natl. Acad. Sci. U.S.A. 103, 17501-17506.

Scalia, F., and Winans, S. S. (1975). The differential projections of the olfactory bulb and accessory olfactory bulb in mammals. J. Comp. Neurol. 161, 31-55.

Schoppa, N. E. (2006). Synchronization of olfactory bulb mitral cells by precisely timed inhibitory inputs. Neuron 49, 271-283.

Shepherd, G. M. (1998). The Synaptic Organization of the Brain. New York, Oxford University Press.

Shepherd, G. M., and Haberly, L. B. (1970). Partial activation of olfactory bulb: analysis of field potentials and topographical relation between bulb and lateral olfactory tract. J. Neurophysiol. 33, 643-653.

Shingo, T., Gregg, C., Enwere, E., Fujikawa, H., Hassam, R., Geary, C., Cross, J. C., and Weiss, S. (2003). Pregnancystimulated neurogenesis in the adult female forebrain mediated by prolactin. Science 299, 117-120.

Wesson, D. W., Carey, R. M., Verhagen, J. V., and Wachowiak, M. (2008). Rapid encoding and perception of novel odors in the rat. PLoS Biol. 6, e82. doi:10.1371/journal.pbio.0060082

Whitman, M. C., and Greer, C. A. (2007). Synaptic integration of adult-generated olfactory bulb granule cells: basal axodendritic centrifugal input precedes apical dendrodendritic local circuits. J. Neurosci. 27, 9951-9961.

Winner, B., Cooper-Kuhn, C. M., Aigner, R., Winkler, J., and Kuhn, H. G. (2002). Long-term survival and cell death of 
newly generated neurons in the adult rat olfactory bulb. Eur. J. Neurosci. 16, 1681-1689.

Yamaguchi, M., and Mori, K. (2005). Critical period for sensory experiencedependent survival of newly generated granule cells in the adult mouse olfactory bulb. Proc. Natl. Acad. Sci. U.S.A. 102, 9697-9702.

Zhang, R., Zhang, Z., Zhang, C., Zhang, L., Robin,A., Wang, Y., Lu, M., and Chopp,
M. (2004). Stroke transiently increases subventricular zone cell division from asymmetric to symmetric and increases neuronal differentiation in the adult rat. J. Neurosci. 24, 5810-5815.

Conflict of Interest Statement: The authors declare that the research was conducted in the absence of any commercial or financial relationships that could be construed as a potential conflict of interest.
Received: 21 September 2009; paper pending published: 18 October 2009; accepted: 28 October 2009; published online: 16 November 2009

Citation: Valley MT, Mullen TR, Schultz LC, Sagdullaev BT and Firestein $S$ (2009) Ablation of mouse adult neurogenesis alters olfactory bulb structure and olfactory fear conditioning. Front. Neurosci. 3:51. doi: 10.3389/neuro.22. 003.2009
This article was submitted to Frontiers in Neurogenesis, a specialty of Frontiers in Neuroscience.

Copyright (C) 2009 Valley, Mullen, Schultz, Sagdullaev and Firestein. This is an open-access article subject to an exclusive license agreement between the authors and the Frontiers Research Foundation, which permits unrestricted use, distribution, and reproduction in any medium, provided the original authors and source are credited. 